Daniil A. Loktev - Alexey A. Loktev - Alexandra V. Salnikova - Anna A. Shaforostova*

\title{
DETERMINATION OF THE DYNAMIC VEHICLE MODEL PARAMETERS BY MEANS OF COMPUTER VISION
}

This study is devoted to determining the geometric, kinematic and dynamic characteristics of a vehicle. To this purpose, it is proposed to use a complex approach applying the models of deformable body mechanics for describing the oscillatory movements of a vehicle and the computer vision algorithms for processing a series of object images to determine the state parameters of a vehicle on the road. The model of the vehicle vertical oscillations is produced by means of the viscoelastic elements and the dry friction element that fully enough represent the behavior of the sprung masses. The introduced algorithms and models can be used as a part of a complex system for monitoring and controlling the road traffic. In addition, they can determine both the speed of the car and its dynamic parameters and the driving behavior of the individual drivers.

Keywords: vehicle, mode of vertical oscillations, Kelvin-Voight and Maxwell viscoelastic elements, image processing, feature point method, Haar primitives, errors of the first and second kind, car motion parameters

\section{Introduction}

At the present time, automobile transport systems, including infrastructure, roads and vehicles, as well as various automated control and monitoring systems, are under active development. The prospects of the transport networks that must be taken into consideration include a steadily increasing traffic speed (and this applies both to the maximum and the average values of movement speed in certain sections of the roads), greater axle loads and vehicle weight, as well as an overall growth in the number of automobiles. An important component of the safe traffic is the timely determination of parameters of the vehicle movement (speed, direction of movement, position at a particular moment of time relative to the roadway and to the other traffic participants) and their type. Timely detection of deviations from the generally accepted traffic trajectories, together with the critical distances between vehicles, as well as a possible overloading of an equipped vehicle or a road train will largely prevent the occurrence of emergencies or catastrophes. To this purpose, it is useful to monitor the position and the behavior of vehicles in some of the most dangerous places only or in the neighborhood of the certain transport objects, because the total control of the crew's movements is difficult enough and the processing of the information thus received is a rather complex computational task.

For such a monitoring, it is proposed to use passive methods for determining the state and traffic parameters of vehicles, by analyzing a series of images of the objects positioned on the roadway. While solving the problem of engineering passive methods for determining the object's geometrical and kinematic parameters, it is important that these methods would allow applying other methods that increase overall accuracy and reliability and reduce operating costs. The last task should be addressed by use of the mobile diagnostic tools, combining various functions of the mobile device, using the integrated controls with the binding of the monitoring results to the one coordinate of the roadway. Besides, it is intended to increase the speed of the diagnostics up to the level of the established speeds of the vehicles at the sections of the roads subject to control.

In the present study, a method is proposed for determining the vehicle parameters based on the procedures of the object's recognition by color and spatial characteristics by means of computer vision.

\section{The simplest models of vertical oscillations}

Modern vehicles can be classified by purpose, patency, wheel formula, the number of axles, climatic design, structural requirements, axle load, load-bearing capacity, as well as by type and distance of transportation. When organizing the line traffic on roads with its optimal loads, all vehicles should have the same traction-speed characteristics, the same acceleration and deceleration dynamics, regardless of the weight of the loaded car [1-2].

When determining the parameters of motion and condition of the vehicle, one must start with the elements whose parameters are known, for example, horizontal and vertical road markings. To study the dynamic behavior of the vehicle, especially pronounced in transient modes of motion, the most interesting are vertical oscillations, including a series of object images obtained at small time intervals that are easier to track with the help of passive methods of processing [3-4].

When constructing a model of the vehicle with which it is possible to determine parameters of the movement, it is proposed to follow from simple to complex, gradually increasing the number

\footnotetext{
* ${ }^{1}$ Daniil A. Loktev, ${ }^{2}$ Alexey A. Loktev, ${ }^{3}$ Alexandra V. Salnikova, ${ }^{4}$ Anna A. Shaforostova

${ }^{1}$ Department of Information Systems and Telecommunications, Bauman Moscow State Technical University, Moscow, Russia

${ }^{2}$ Department of Transport Construction, Russian University of Transport (MIIT), Moscow, Russia

${ }^{3}$ International Laboratory of Statistics of Stochastic Processes and Quantitative Finance of Tomsk State University, Russia and Department

of Management Systems and Information Technologies in Building, Voronezh State Technical University, Russia

${ }^{4}$ Department of Radio Engineering, Voronezh State Technical University, Russia

E-mail: amicus.lat@yandex.ru
} 


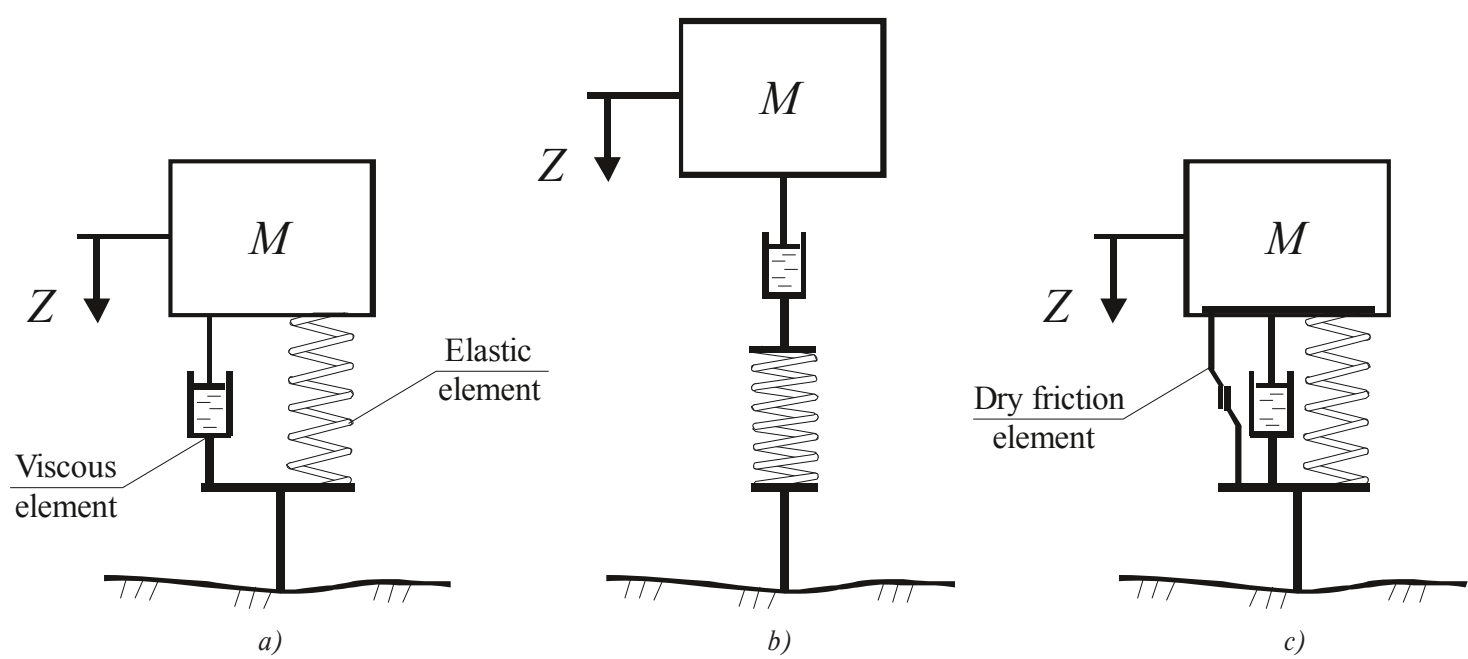

Figure 1 Model of the vehicle with one degree of freedom, with viscoelastic elements that simulate the dynamic interaction of the vehicle and the road surface: a) Kelvin-Voigt element, b) Maxwell element, c) Kelvin-Voigt element with dry friction

of defined characteristics and factors to be taken into account. In the beginning, to describe the vertical vibrations of the vehicle, one can choose a system with a single degree of freedom, the mass of which expresses the mass of a loaded car $M$ (Figures 1a, $1 \mathrm{~b}$ ), as well as the system with two degrees of freedom (Figure 1c). Then, the lower mass determines the reduced mass of the wheel pair $m$, and the upper mass of the vehicle body and its load $M_{1}$. In the first case, the vertical displacement associated with the center of gravity of the system is denoted by $Z$; in the second case the displacement of the wheel pair is denoted by $z_{1}$, and the displacement of the vehicle body is $Z_{1}$. To simulate the interaction of various parts of the vehicle and the roadway, the elastic elements-springs are used, viscous dampers and dissipative dry friction elements. Various combinations of the listed connections make it possible to obtain adequate and accurate models of the dynamic behavior of both individual components, aggregates and elements and the entire vehicle. Figure 1a shows a viscoelastic Kelvin-Voigt element that simulates the interaction of the vehicle and the road surface. In Figure 1b, the Maxwell element is presented, which is a sequential connection of a viscous and elastic element. In Figure 1c, a dry friction element has been added to the Kelvin-Voigt element, which makes it possible to simulate springs of the vehicle more fully $[1,2,5]$.

There is also a model of interaction of a vehicle with the road surface with the two degrees of freedom, a separate body with its mass; the wheel pair is represented by geometrical and mechanical parameters. This representation allows one to separately simulate the interaction between the body of a vehicle and a wheel pair, as well as the properties of springs, by introducing a dry-friction element into the system responsible for rheological properties of the suspension. The lower viscoelastic element of the Kelvin-Voight type allows simulating the interaction of the wheel pair and the roadway, and in the future it will allow taking into account various defects of the road surface.

The choice of a specific mathematical model for the vehicle depends on a number of factors. They include the type of the vehicle; the parameters determined and the assumptions made concerning, for example, the continuity of the movement, being either rectilinear or even. In this case, the theory of small oscillations applicable to the system of bodies and the presupposition that springs and the wheels of the vehicle have only vertical compliance are taken into account, while the horizontal forces of inertia of wheel pairs are not [1, 3, 6].

When describing vertical oscillations of models Figure 1a and Figure 1b, it is assumed that viscoelastic elements do not lose stability, i.e. points of the vehicle's body and the wheel in the area of contact with the road surface can move rectilinearly along the same straight line.

In this paper is proposed to consider the joint movement of the vehicle body and the wheel pair without taking into account those impacts that are actually present from the roadway and moving cargo. The movement of the two interacting bodies (the body of the vehicle and the wheel pair) after the start of the contact for the model of viscoelastic body of the Kelvin-Voigt type (Figure 1a), with respect to the fixed reference system, is described by a system of the second-order differential equations:

$M_{1}\left(\ddot{z}_{1}+\ddot{z}_{3}\right)+K\left(\dot{z}_{1}-\dot{z}_{2}\right)+C\left(z_{1}-z_{2}\right)=0$,
$m_{1}\left(\ddot{z}_{2}+\ddot{z}_{3}\right)+K\left(\dot{z}_{2}-\dot{z}_{1}\right)+C\left(z_{2}-z_{1}\right)=0$,

if one takes the Maxwell's viscoelastic element as the basis of the buffer model simulating a wide range of vehicle suspension properties, then the system of determining equations will take the form:

$$
\begin{aligned}
& M_{1}\left(\ddot{z}_{1}+\ddot{z}_{3}\right)-K \int_{0}^{t}\left(\dot{z}_{1}-\dot{z}_{2}\right) \exp \left(-\frac{t-t^{\prime}}{\tau_{1}}\right) d t^{\prime}+ \\
& +C\left(z_{1}-z_{2}\right)=0, \\
& m_{1}\left(\ddot{z}_{2}+\ddot{z}_{3}\right)-K \int_{0}^{t}\left(\dot{z}_{2}-\dot{z}_{1}\right) \exp \left(-\frac{t-t^{\prime}}{\tau_{1}}\right) d t^{\prime}+ \\
& +C\left(z_{2}-z_{1}\right)=0,
\end{aligned}
$$

where $M_{1}$ and $m_{1}$ are the weights of the body and wheel pairs, respectively; $z_{1}$ and $z_{2}$ are the coordinates of the center of gravity 


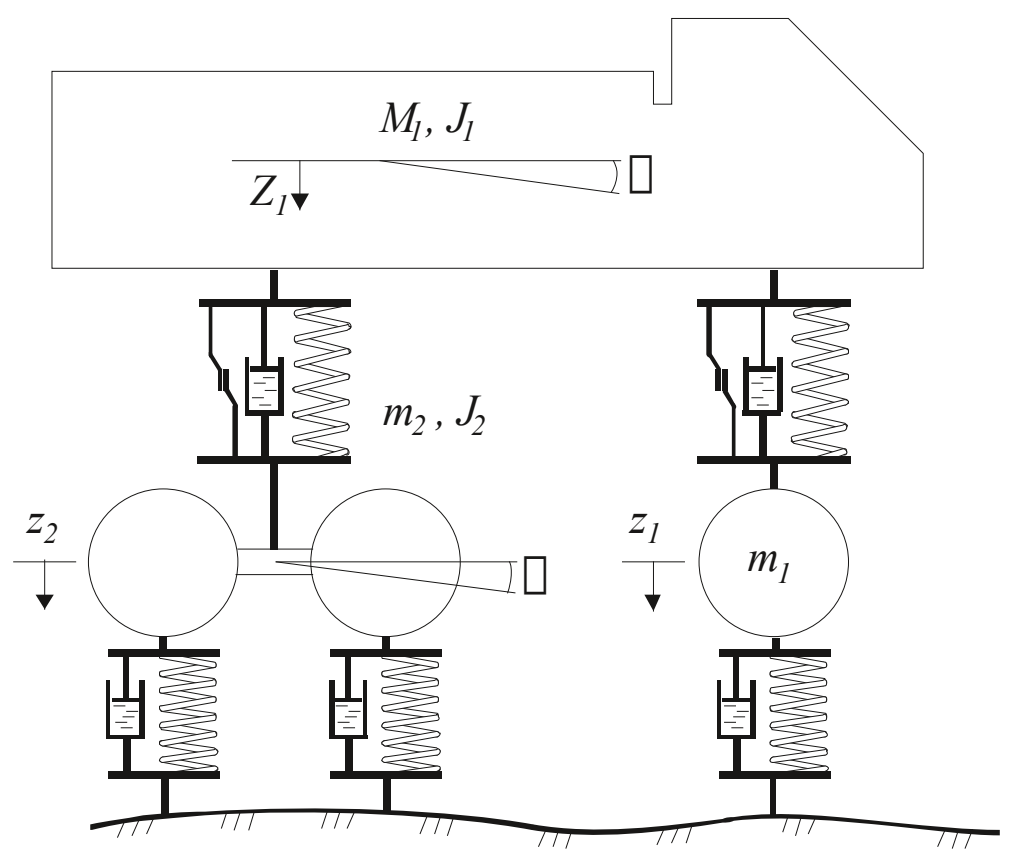

Figure 2 Flat model of a three-axle car with five degrees of freedom

of the body and the wheel pair relative to the upper point of the buffer, respectively; $z_{3}$ is the coordinate of the upper point of the buffer relative to the inertial reference frame; $K$ is the coefficient of the viscous resistance of the buffer simulating the interaction of the vehicle and the road surface; $C$ is the coefficient of rigidity of the elastic element in the buffer.

The system of defining equations (1) or (2) depending on the selected model must be supplemented with the initial conditions characterizing the state of the system of bodies at the initial time $t=0$ :

$z_{1}(t=0)=0, \quad z_{2}(t=0)=0$

$\dot{z}_{1}(t=0)=0, \quad \dot{z}_{2}(t=0)=0$.

To evaluate the interaction of the body of the vehicle, the wheel pair and the roadway, it is proposed to use three characteristics (power, geometrical and kinematic):

- the force of interaction between various sprung parts of the system $P(t)$, taking into account the influence of linear resistance, elastic forces and dry friction; in some cases, the greatest interest is the maximum value of this force $\max _{t \in[0, \infty)} P(t)$;

- the vertical movement of the vehicle's body $z(t)$, including its maximum value $\max _{t \in[0, \infty)} z(t)$;

- the vertical acceleration of the vehicle points as one of the main criteria for the smooth running of the vehicle $\max _{t \in[0, \infty)} \ddot{z}(t)$.

Depending on the type of the vehicle, more complex models can be used to describe the operation of the main components, assemblies, vehicle structures and their elements, as well as the behavior of cargos, driver and passengers. That will allow a more accurate study of the dynamic behavior and state of the vehicle under the different traffic conditions and depending on the quality of the road surface $[1,4]$.

\section{Plane model of a vehicle with three wheel axles}

In addition to the simplest models shown in Figure 1, in this paper is considered a flat model in the form of a system of solids (vehicle's body and wheel pairs), connected with each other and with the roadway by the viscoelastic elements with the elements of dry friction at the places of suspension of weights. In fact, those elements are elastic-dissipative bonds that are responsible for the model of interaction of various elements with each other and taking into account the rheological properties of specific materials and structural elements.

For accurate estimation of the computational models of a vehicle, it is necessary to carry out the frequency analysis of their own and forced oscillations, as well as to study the behavior of vehicle's structures in various design situations and under different driving conditions $[2,3,5]$.

This study also considers the design of a three-axle vehicle with the five degrees of freedom (Figure 2). As the generalized coordinates the following ones are accepted: $Z$-vertical movement of the vehicle's body (center of gravity); $\varphi$ - angular movement of the vehicle's body; $z_{1}$ - vertical movement of the first axis of the car; $z_{2}$ - reduced vertical movement of the second axle of the vehicle, in fact, this is a joint movement of the 2 nd and 3rd bridges, connected to each other and representing a trolley; $\psi$ - angular displacement of dual axles of the vehicle.

Functional equations describing the movement of the vehicle in differential form can be obtained in terms of the d'Alembert principle:

$M \ddot{Z}(t)+F_{1}\left(v_{1}, \dot{v}_{1}\right)+F_{2}\left(v_{2}, \dot{v}_{2}\right)-M_{g}=0$,

$J_{1} \ddot{\varphi}(t)+F_{1}\left(v_{1}, \dot{v}_{1}\right) a_{T}-F_{2}\left(v_{2}, \dot{v}_{2}\right) b_{T}=0$,

$m_{1} \ddot{z}_{1}(t)-F_{1}\left(v_{1}, \dot{v}_{1}\right)+R_{1}\left(u_{1}, \dot{u}_{1}\right)-m_{1} g=0$,

$m_{2} \ddot{z}_{2}(t)-F_{2}\left(v_{2}, \dot{v}_{2}\right)+R_{2}\left(u_{2}, \dot{u}_{2}\right)+R_{3}\left(u_{3}, \dot{u}_{3}\right)-m_{2} g=0$,

$J_{2} \ddot{\psi}(t)+R_{2}\left(u_{2}, \dot{u}_{2}\right) l_{2} / 2-R_{3}\left(u_{3}, \dot{u}_{3}\right) l_{2} / 2=0$, 
where $F_{i}\left(v_{i}, \dot{v}_{i}\right)$ and $R_{i}\left(u_{i}, \dot{u}_{i}\right)$ are the viscous resistance forces in a buffer that simulates the interaction of the body and the $i$-th wheel pair; $v_{i}=v_{i}(t), \dot{v}_{i}=\dot{v}_{i}(t)$ are the vertical displacement and the speed of the buffer deformation over the $i$-th wheel pair, respectively; $u_{i}=u_{i}(t), \dot{u}_{i}=\dot{u}_{i}(t)$ are the vertical displacement and the wheel deformation speed related to the $i$-th axis, respectively.

The defining relations for the forces $F_{i}$ and $R_{i}$ can be represented taking into account the terms responsible for the dissipative properties of the buffer including the viscous resistance according to the Kelvin-Voigt model and dry sliding friction according to the Coulomb model:

$$
\begin{aligned}
& F_{i}\left(v_{i}, \dot{v}_{i}\right)=F_{i}\left(v_{i}\right)+k_{p i} \dot{v}_{i}+T_{p i} \operatorname{sign}\left(\dot{v}_{i}\right), \\
& R_{i}\left(u_{i}, \dot{u}_{i}\right)=R_{i}\left(u_{i}\right)+k_{s i} \dot{u}_{i},
\end{aligned}
$$

where $k_{p i}, k_{s i}$ are the damping coefficients of the viscoelastic buffer and the $i$-th axis of the wheel and $T_{p i}$ is the coefficient of dry friction in the buffer over the $i$-th axis. The functions $F_{i}\left(v_{i}\right)$ and $R_{i}\left(u_{i}\right)$ usually take a piecewise linear exponential form $[2,5]$.

To reduce the number of unknown factors in the functional equations and the desired functions, one can represent the vertical displacements of axes of wheel pairs $v_{i}$ and $u_{i}$ through the generalized coordinates. Then, the parameters of the road profile are taken into account, allowing specifying the initial movements, for example, when the vehicle goes over the element of forced reduction of the vehicle speed («speed bump»).

\section{Method for determining the motion parameters by analyzing images}

To experimentally determine geometrical and kinematic characteristics of the vehicle's movement both in the horizontal and the vertical plane (direction of movement, speed and distance to the desired object), it is necessary to recognize the vehicle type, as well as to detect the number of wheel axes. Determining the movement parameters of the vehicle's body and its wheels can be carried out when considering rotational motion (wheels) and translational motion (body) [7-9].

At the stage of the primary image processing, the characteristic points of the image are distinguished, and the optical flow in their vicinity is calculated. In this case, the pixel of the object with the plane and time coordinates $(x, y, t)$ and intensity $I(x, y, t)$ receive increments $(\Delta x, \Delta y, \Delta t)$ during the change of one frame to another [10-12]. The changed pixel intensity can be represented in the form of a Taylor series, given the smallness of the possible displacements:

$$
\begin{aligned}
& I(x+\Delta x, y+\Delta y, t+\Delta t)= \\
& I(x, y, t)+\frac{\partial I}{\partial x} \Delta x+\frac{\partial I}{\partial y} \Delta y+\frac{\partial I}{\partial t} \Delta t .
\end{aligned}
$$

To calculate the optical flow of an image, it is proposed to use the Lucas-Canade method, which is based on the assumption that the optical flow is the same for all the pixels within a small rectangular image area, which includes a certain set of pixels $\left(q_{i}\right)_{i=1}^{n}$ [13]. The components of the optical flow velocity $v=\left[v_{x}, v_{y}\right]^{T}$ for the central pixel $p$ of the set can be obtained by solving the defining system of equations, [14]:

$I_{x}\left(q_{i}\right) v_{x}+I_{y}\left(q_{i}\right) v_{y}+I_{t}=0$

where the fact is taken into account that the intensity depends [15] on the position of each pixel $q_{i}$.

An important task that one faces while solving the system of equations (6) is the choice of the size of a rectangular frame around the examined pixel [16-18]. It concerns with the comparison of the two mutually exclusive conditions: while reducing the frame allows one to separate the moving objects with a fixed background [19], the frame magnification allows one to detect and track large object movements [20]. To solve the dual problem of the Lucas-Canade method implementation, it is proposed to use the Bouguet iterative scheme [21-23].

Comparison of the two images of a video sequence can be represented as the following sequence of actions:

Step 1. Inputting images $I, J$ and the coordinates of the examined pixel $u$ into the image $I$.

Step 2. Creating interactive diagrams of the source images $\left\{I^{L}\right\}_{L=0 \ldots L m}$ and $\left\{J^{L}\right\}_{L=0 \ldots L m}$.

Step 3. Initializing the offset accumulated by the passed levels: $g^{L m}=\left[\begin{array}{l}0 \\ 0\end{array}\right]$.

Step 4. Cycling through $L$ levels from $L_{m}$ to 0 with the decrement -1 .

Step 5. Calculating the coordinates $\left(p_{x}, p_{y}\right)$ of the pixel $u$ at the image $I^{L}: u^{L}=\frac{u}{2^{L}}=\left[\begin{array}{c}p_{x} \\ p_{y}\end{array}\right]$

Step 6. Calculating the derivatives of the image $I^{L}$ at the spatial coordinates: $I_{x}(x, y)=\left\{I^{L}(x+1, y)-I^{L}(x-1, y)\right\} / 2$.

Step 7. Calculating the gradient matrix in the image plane: $G=\sum_{x=p_{x}-w_{x}}^{p_{x}+w_{x}} \sum_{y=p_{y}-w_{y}}^{p_{y}+w_{y}}\left[\begin{array}{cc}I_{x}^{2}(x, y) & I_{x}(x, y) I_{y}(x, y) \\ I_{x}(x, y) I_{y}(x, y) & I_{x}^{2}(x, y)\end{array}\right]$.

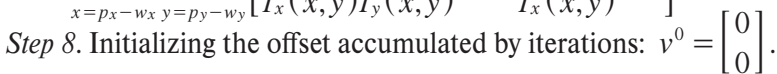

Step 9. Subcycling of iterations $k$ from 1 to 5 .

Step 9.1. Image subtraction (evaluation of the time derivative of the image): $\delta I_{k}(x, y)=I^{L}(x, y)-J^{L}\left(x+g_{x}^{L}+v_{x}^{k-1}, y+g_{y}^{L}+v_{y}^{k-1}\right)$.

Step 9.2. Calculating the vector differences: $b_{k}=\sum_{x=p_{x}-w_{x}}^{p_{x}+w_{x}} \sum_{y=p_{y}-w_{y}}^{p_{y}+w_{y}}\left[\begin{array}{l}\delta I_{k}(x, y) I_{x}(x, y) \\ \delta I_{k}(x, y) I_{y}(x, y)\end{array}\right]$.

Step 9.3. Optical flow calculation: $\eta^{k}=G^{-1} b_{k}$, where $G=A^{T} A=\sum_{i=1}^{n}\left[\begin{array}{cc}I_{x}^{2}\left(q_{i}\right) & I_{x}\left(q_{i}\right) I_{y}\left(q_{i}\right) \\ I_{x}\left(q_{i}\right) I_{y}\left(q_{i}\right) & I_{y}^{2}\left(q_{i}\right)\end{array}\right]$ is the matrix of moments of the second order of the rectangular area of the image; $b=\sum_{i=1}^{n}\left[\begin{array}{l}-I_{t}\left(q_{i}\right) I_{x}\left(q_{i}\right) \\ -I_{t}\left(q_{i}\right) I_{y}\left(q_{i}\right)\end{array}\right]$ is the vector of the mixed multiplication.

Step 9.4. Updating the offset accumulated by iterations: $v^{k}=v^{k-1}+\eta^{k}$.

Step 10. Calculating the offset for level $L: d^{L}=v^{k}$.

Step 11. Calculating the offset accumulated up to the next level $L-1: g^{L-1}=2\left(g^{L}+d^{L}\right)$.

Step 12. Calculating the total offset: $d=g^{0}+d^{0}$. 


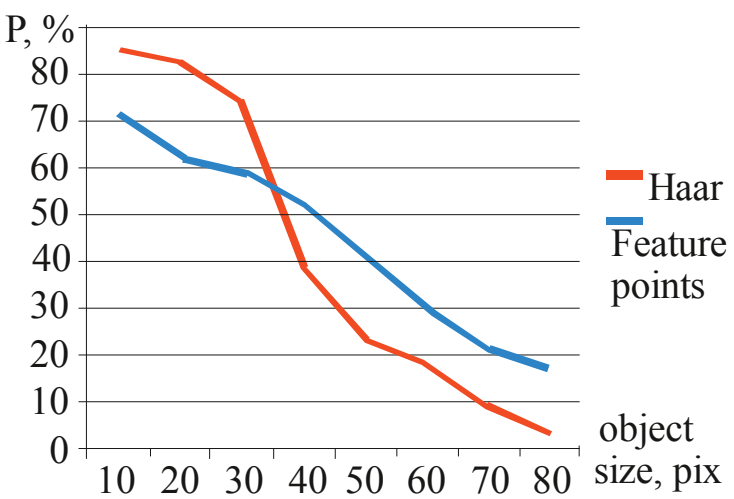

a)

Figure 3 The dependence of the error probability of the first (a) and second (b) kinds upon the object's size

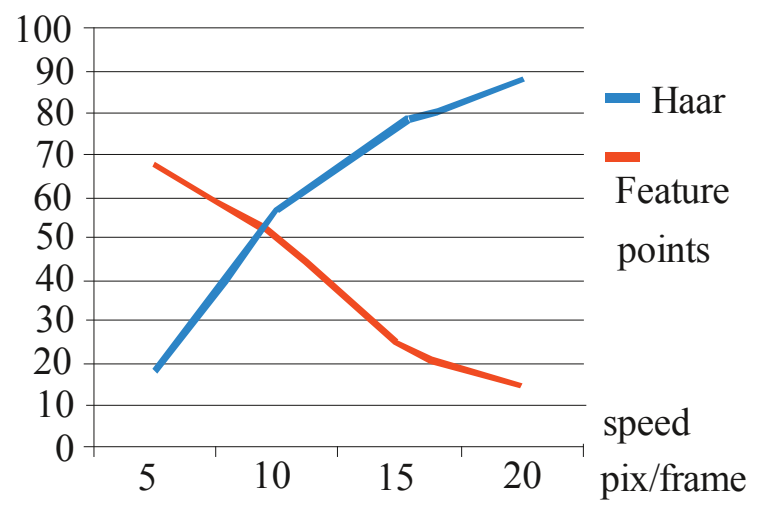

a)

Figure 4 Dependence of the error probability of the first (a) and second (b) kinds upon the object's speed
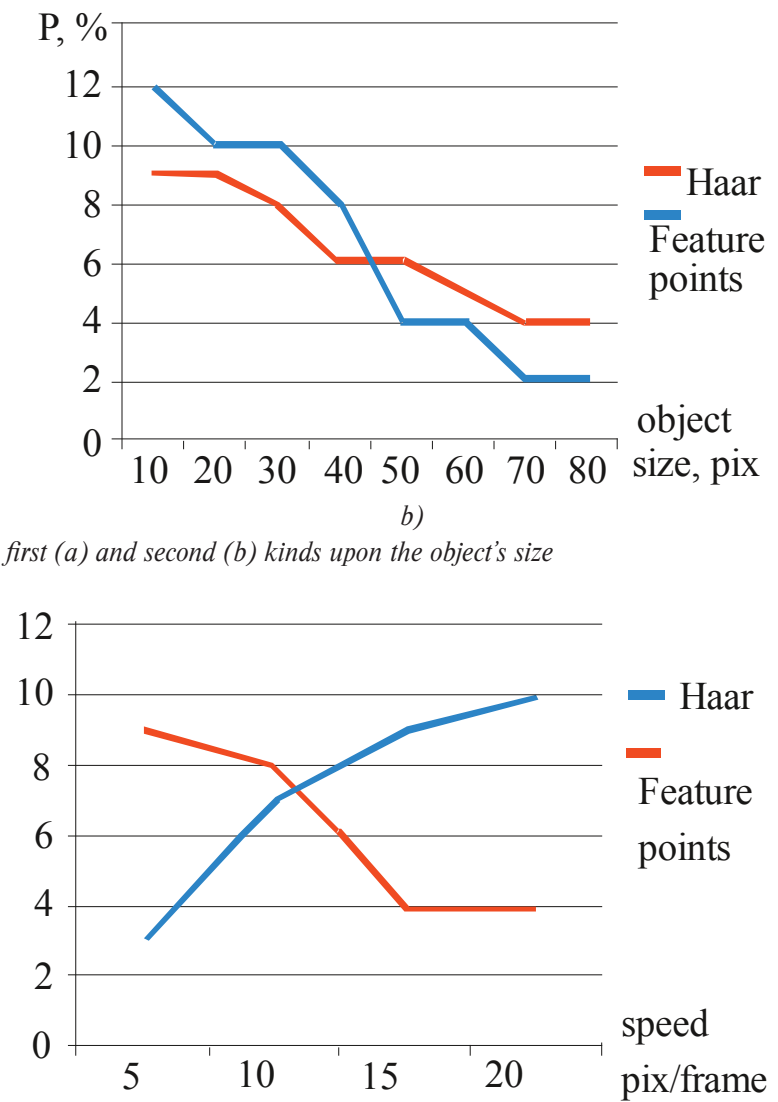

b)
Step 13. Calculating the coordinates of the examined pixel on the image $J: w=u+d$.

Step 14. Outputting the coordinates of the pixel $w$ on to the image $J$.

The described algorithm can be used to determine, for the last $N$ frames, the movements of the center of the selected frame relative to the background in the pixels $\Delta X_{o b}, \Delta Y_{o b}$ which is necessary to determine the velocities of object points [19, 24].

Having a priori camera information - the height $H_{f r}$ and the width $W_{f r}$ of the frame, the angles in the radians $\alpha_{f r}$ and $\beta_{f r}$, vertically and horizontally, - one can find the actual horizontal and vertical offsets of the object from the proportions of the right-angled triangles that define the positions of the image and the real object:

$$
\begin{aligned}
& \frac{\Delta \alpha_{o b}}{\alpha_{f r}}=\frac{\Delta Y_{o b}}{H_{f r}} \Rightarrow \Delta \alpha_{o b}=\frac{\Delta Y_{o b}}{H_{f r}} \alpha_{f r}, \\
& \tan \Delta \alpha_{o b}=\frac{\Delta y}{l} \Rightarrow \Delta y=l \Delta \alpha_{o b}=\frac{\alpha_{f r} \Delta Y_{o b}}{H_{f r}} l .
\end{aligned}
$$

Estimations of the velocity components $v_{x}, v_{y}$ are obtained by dividing the offsets by the time interval $N \tau$ ( $\tau$ - frame duration):

$v_{x}=\frac{\Delta x}{N \tau}=\frac{\beta_{f r} \Delta X_{o b}}{W_{f r}} \frac{l}{N \tau}, v_{y}=\frac{\Delta y}{N \tau}=\frac{\alpha_{f r} \Delta Y_{o b}}{H_{f r}} \frac{l}{N \tau}$

When determining the distance to the vehicle, the height of the frame of the object $H_{o b}$ in pixels [25-27] is referred to. Assuming that the vertical viewing angle of the camera in the radians is $\alpha_{f r}$, the frame height in pixels is $H_{f r}$, the real height of the object in meters is $h$, it is possible to determine the actual distance to the object in meters that is $l$.

The angle at which the object is visible is determined by the proportion: $\alpha_{o b}=H_{o b} \alpha_{f r} / H_{f r}$.

Then the actual distance to the object, taking into account the small value of the viewing angle, is the following: $l=h / \alpha_{o b}=H_{f r} h / \alpha_{o b} H_{o b}$.

The estimation of the velocity projection $v_{z}$ of the object is made by changing the height of the object during the time $N_{\tau}$, then:

$l(t)=\frac{H_{f r}}{\alpha_{f r} H_{o b}(t)} h$,

$l(t-N \tau)=\frac{H_{f r}}{\alpha_{f r} H_{o b}(t-N \tau)} h$,

$v_{z}=\frac{l(t)-l(t-N \tau)}{N \tau}=$

$$
=\left[\frac{H_{f r}}{H_{o b}(t)}-\frac{H_{f r}}{H_{o b}(t-N \tau)}\right] \frac{h}{\alpha_{f r} N \tau} .
$$

The actual speed of the object is calculated by the geometrical sum of the projections: $v=\sqrt{v_{x}^{2}+v_{y}^{2}+v_{z}^{2}}$. Knowing the speed function, one can determine the acceleration of the vehicle and its projection on different coordinate axes. 


\section{Numerical investigation}

In addition to the Lucas-Canade method, the Adaptive Boosting algorithm has been considered [28-30]. Probabilities of errors of the first and second kind have been determined [31-33], depending on the object's size at the image (Figure 3a, 3b) and on the object's speed (Figure 4a, 4b)

Figures 3 and 4 show that the for larger objects (more closely located) and for the slower objects the possibility of errors of the first and the second kind is less when the algorithm of the object recognition using the Haar primitives is applied, while for all the other objects, application of the algorithm, using the feature points, is the most suitable. Thus, to reduce the probability of errors during the recognition of the objects, it is proposed to use only two parameters for the initial recognition of the object: the distance from the camera and its speed. It is assumed that the speed of the objects can belong to one of the two groups - "fast" or "slow", and the distance to them - to the "far" or "close" groups. Establishing the boundaries of the definition of these groups is performed by means of the statistical analysis tools.

As a result, when determining the speeds of the vertical and horizontal movement of the vehicle, as well as the vertical linear and angular accelerations, the weight of the vehicle and the weights of the individual parts of the vehicle, reduced to the wheel axles can be determined. This will contribute to the control of the road congestion, the traffic density at the individual sections, as well as toe compliance with the regulatory requirements of the cargo transportation and traffic rules.

\section{Conclusion}

Algorithms, methods and models introduced in this study can be implemented as a part of an integrated hardware-software system for monitoring and controlling the motion that can allow one to determine the geometrical (movement, position), kinematic (speed, path) and dynamic (weight, acceleration) motion parameters of the car. As the primary information on the object is proposed to be obtained through its image in the optical range, this method is not associated with the additional electromagnetic radiation and thus it is not dangerous for living organisms. It is also rather more difficult to be detected and thus it is safer against possible counter detection systems. The conducted numerical studies, based on the developed algorithms and models, demonstrate the better occurrence values of errors of the first and the second kind during the system operation depending on the speed and size of the object being measured. The presented detection scheme can operate in the real time during its computerbased implementation.

\section{Acknowledgement}

This study was financially supported by the Russian Science Foundation (research project No. 17-11-01049).

\section{References}

[1] AZIMOV, S. Z., LEBEDEV, O. V., SHERMUKHAMEDOV, A. A. Optimization of calculation of the process of braking of wheel machines (in Russian). 8th Russian Congress of Theoretical and Applied Mechanics : proceedings. 2001, p. 211.

[2] SOUKUP, J., SKOCILAS, J., SKOCILASOVA, B. Vertical vibration of the vehicle model with higher degree of freedom. Procedia Engineering [online]. 2014, 96, p. 435-443. ISSN 1877-7058. Available from: https://doi.org/10.1016/j.proeng.2014.12.113

[3] DEVYATERIKOV, E. A., MIKHAILOV, B. B. Vision system for measuring the path of a mobile robot (in Russian). Mechanics, Control and Informatics. 2012, 2(8), p. 219-224. ISSN 2075-6836.

[4] ZAMOTAYLOV, O. V. Problems of image recognition of the device of subsurface radar on the basis of mobile road laboratory (in Russian). T-Comm-Telecommunications and Transport. 2010, 6, p. 38-42. ISSN 2072-8735, eISSN 2072-8743.

[5] LOKTEV D. A. Determination of object parameters by a series of its images in the integrated monitoring system (in Russian). Path and Track Facilities. 2015, 2, p. 31-33. ISSN 0131-5765

[6] SUN, Z., BEBIS, G., MILLER, R. On-road vehicle detection using optical sensors: A review. 7th International IEEE Conference on Intelligent Transportation Systems : proceedings [online]. 2004. ISBN 0-7803-8500-4. Available from: https://doi.org/10.1109/ITSC.2004.1398966

[7] BEDER, C., BARTCZAK, B., KOCH, R. A comparison of PMD-cameras and stereo-vision for the task of surface reconstruction using patchlets. 2007 IEEE Conference on Computer Vision and Pattern Recognition : proceedings [online]. Vol. 4. IEEE Service Center : Piscataway, NJ, 2007. ISBN 1-4244-1179-3, eISBN 1-4244-1180-7, p. 17-22. Available from: https://doi.org/10.1109/CVPR.2007.383348

[8] WIEDEMANN, M., SAUER, M., DRIEWER, F., SCHILling, K. Analysis and characterization of the PMD camera for application in mobile robotics. 17th World Congress - International Federation of Automatic Control : proceedings [online]. 2008. ISBN 978-1-1234-7890-2. Available from: https://doi.org/10.3182/20080706-5-KR-1001.3304

[9] LITOMISKY, K. Consumer RGB-D cameras and their applications [online]. Ph.D. Thesis. University of California: Riverside, 2012. Available from: http://alumni.cs.ucr.edu/ $\sim$ klitomis/files/RGBD-intro.pdf

[10] HAHNE, U. Real-time depth imaging [online]. Ph.D. Thesis. Technische Universitat Berlin: Berlin, 2012. Available from: https://d-nb.info/1023762218/34 
[11] GIL, P., POMARES, J., TORRES, F. Analysis and adaptation of integration time in PMD camera for visual serving. 20th International Conference on Pattern Recognition : proceedings [online]. 2010. ISSN 1051-4651. Available from: https://doi.org/10.1109/ICPR.2010.85

[12] SINGH S., WEST J. Cyclone: A laser scanner for mobile robot navigation. Technical Report CMU-RI-TR-91-18. The Robotics Institute, Carnegie Mellon University: Pittsburgh, 1991.

[13] DANKO, M., TARABA, M., ADAMEC, J., DRGONA, P. Visualization of Scoda instrument cluster. Communications Scientific Letters of the University of Zilina [online]. 2018, 20(1), p. 27-31. ISSN 1335-4205, eISSN 2585-7878. Available from: http://komunikacie.uniza.sk/index.php/communications/article/view/39

[14] VELAS, A., LOVECEK, T., VALOUCH, J., DWORZECKI, J., VNENCAKOVA, E. Testing radio signal range of selected components. Communications - Scientific Letters of the University of Zilina [online]. 2018, 20(2), p. 68-77. ISSN 1335-4205, eISSN 2585-7878. Available from: http://komunikacie.uniza.sk/index.php/communications/article/view/90

[15] BOROS, M., SISER, A., KEKOVIC, Z., MAZAL, J. Mechanical characteristics of cylinder pin tumbler locks as they relate to resistance testing. Communications - Scientific Letters of the University of Zilina [online]. 2018, 20(2), p. 96-101. ISSN 1335-4205, eISSN 2585-7878. Available from: http://komunikacie.uniza.sk/index.php/communications/article/view/94

[16] BENDER, C., DENKER, K., FRIEDRICH, M., HIRT, K., UMLAUF, G. A hand-held laser scanner based on multi-camera stereomatching. IRTG 1131 - Visualization of Large and Unstructured Data Sets Workshop 2011 : proceedings [online]. 2011. Available from: https://doi.org/10.4230/OASIcs.VLUDS.2011.123

[17] DESCHENES, F., ZIOU, D. Depth from defocus estimation in spatial domain. Computer Vision and Image Understanding [online]. 2001, 81(2), p. 143-165. ISSN 1077-3142. Available from: https://doi.org/10.1006/cviu.2000.0899

[18] RAJABZADEH, T., VAHEDIAN, A., POURREZA, H. R. Static object depth estimation using defocus blur levels features. 6th International Conference on Wireless Communications Networking and Mobile Computing : proceedings. 2010. ISBN 978-1-4244-3709-2.

[19] BORACHI, G., CAGLIOTI, V. Motion blur estimation at corners. 3rd International Conference on Computer Vision Theory and Applications : proceedings. 2008. ISBN 978-989-8111-21-0.

[20] ZHUO, S., SIM, T. Defocus map estimation from a single image. Pattern Recognition [online]. 2011, 44(9), p. $1852-1858$. ISSN 0031-3203. Available from: https://doi.org/10.1016/j.patcog.2011.03.009

[21] ELDER, J. H., ZUCKER, S. W. Local scale control for edge detection and blur estimation. IEEE Transaction on Pattern Analysis and Machine Intelligence [online]. 1998, 20(7), p. 120-127. ISSN 0162-8828, eISSN 1939-3539. Available from: https://doi.org/10.1109/34.689301

[22] LOSCH, S. Depth from blur combining image deblurring and depth estimation. Bachelor's Thesis. Saarland University: Homburg, 2009.

[23] LIN, H.-Y., CHANG, C.-H. Depth from motion and defocus blur. Optical Engineering [online]. 2006, 45(12), p. 127201-1- 127201-12. ISSN 0091-3286, eISSN 1560-2303. Available from: https://doi.org/10.1117/1.2403851

[24] ALEXIEV K., NIKOLOVA I., ZAPRYANOV G. 3D scenes recovery through an active camera based on blur assessment of the resulting image. Information Technologies and Control. 2008, 3-4, p. 10-20. ISSN 1392-124X, eISSN 2335-884X.

[25] LOKTEV, D. A., ALFIMTSEV, A. N., LOKTEV, A. A. Modeling of an integrated system of video monitoring inside the building. Part 2. The Algorithm of Recognition of Objects (in Russian). Vestnik MGSU. 2012, 5, p. 124-131. ISSN 1997-0935, eISSN 2304-6600.

[26] LUCAS, B. D., KANADE, T. An iterative image registration technique with an application to stereo vision. 7th International Joint Conference on Artificial Intelligence : proceedings. 1981.

[27] BOUGUET J.-Y. Pyramidal implementation of the lucas-kanade feature tracker. Intel Corporation Microprocessor Research Labs: Santa Clara, CA, USA, 2000.

[28] HARRIS, C., STEPHENS, M. A combined corner and edge detector. 4th Alvey Vision Conference : proceedings [online]. 1988. Available from: https://doi.org/10.5244/C.2.23

[29] LOKTEV, A. A., SYCHEV, V. P., LOKTEV, D. A., DMITRIEV, V. G. Automated system for identifying defects in wheels of the rolling stock on the basis of assessment of shock nonaxisymmetric impact of wheels on rail when modeling the track structure with orthotropic plate (in Russian). Engineering and Automation Problems. 2017, 4, p. 59-70. ISSN 0234-6206.

[30] VIOLA P., JONES M. J. Robust real-time object detection. Second Workshop on Statistical and Computational Theories of Vision: procedings. 2001.

[31] CHERNOYAROV, O. V., VACULIK, M., SHIRIKYAN, A., SALNIKOVA, A. V. Statistical analysis of fast fluctuating random signals with arbitrary-function envelope and unknown parameters. Communications - Scientific Letters of the University of Zilina [online]. 2015, 17(1A), p. 35-43. ISSN 1335-4205, eISSN 2585-7878. Available from: http://komunikacie.uniza.sk/index.php/ communications/article/view/410

[32] LELEGARD, L., VALLET, B., BREDIF, M. Multiscale haar transform for blur estimation from a set of images. ISPRS Conference PIA 2011. International Archives of Photogrammetry, Remote Sensing and Spatial Information Science : proceedings [online]. 2011. Available from: https://doi.org/10.5194/isprsarchives-XXXVIII-3-W22-65-2011

[33] LOKTEV, D. A., LOKTEV A. A. Determination of object location by analyzing the image blur. Contemporary Engineering Sciences [online]. 2015, 8(9), p. 467-475. ISSN 1313-6569, eISSN 1314-7641. Available from: http://dx.doi.org/10.12988/ces.2015.52198 